\title{
Bemerkungen zu drei thebanischen Statuen der Spätzeit
}

\section{Die Familie des Wezirs Hr nach den Statuen Kairo JE 37413 UND JE 37848}

Wenige Jahre nach der Entdeckung der »Cachette « von Karnak hat G. Legrain über zwei der dort gefundenen Statuen berichtet $\left(^{1}\right)$, die offenbar aus einer Familie, von Vater und Sohn, stammen: Die eine $(\mathrm{K} .441=\mathrm{JE}$ 37413) gehört einem Priester namens $N b$-ntrw, Sohn eines ' $n h$-wn-nfr; sie ist von seinem Sohn gestiftet, der wie sein Großvater $n h$-wn-nfr hieß. Die andere $(\mathrm{K} .567=\mathrm{JE}$ 37848) gehört einem Priester $n h$-wn-nfr, Sohn eines $\mathrm{Nb}$-ntrw und Enkel eines weiteren $n h$-wn-nfr. Da auch die Titel sich weitgehend entsprechen, lag es auf der Hand, daß der Besitzer von JE 37848 der Sohn des Besitzers von JE 37413 (und Stifter dieser Statue) ist. Auf beiden Statuen wird der Großvater des $N b$-ntrw bzw. der Urgroßvater des 'nh-wn$n f r$ als Endglied der genealogischen Kette genannt, der offenbar zugleich der ranghöchste Ahnherr der Familie war, nämlich ein Wezir. Allerdings hat Legrain den Namen dieses Wezirs auf JE 37848 als $(H r)$ gelesen, auf JE 37413 dagegen als IN (P3-mjw). Da aber sonst alles dafür sprach, daß die beiden Statuen Vater und Sohn gehören, lag es nahe, P3-mjw einfach zum Beinamen des Wezirs $H r$ zu erklären, und das umso mehr, als ein Wezir P3-mjw tatsächlich auf mehreren anderen Denkmälern belegt ist.

Diese Gleichsetzung der Wezire $H r$ und P3-mjw und die Identifizierung dieser Person mit dem bekannten Wezir P3-mjw ist von A. Weil übernommen worden $\left(^{2}\right)$ und dann v.a. von H. Kees weiter ausgebaut worden $\left({ }^{3}\right)$. Sie würde bedeuten, daß die beiden Statuen noch in die 3. Zwischenzeit gehören, denn der Wezir P3-mjw war ein Generationsgenosse

(1) ASAE 7, 1906, 50-53.

(2) Die Veziere des Pharaonenreiches, Straßburg 1908, 134-135.

(3) Das Priestertum im ägyptischen Staat, PÄ 1, 1953, 229-230; »Beiträge zur Geschichte der thebanischen Vezirsfamilie Pimui«, ASAE 54, 1956, 141-148. 
Osorkons III. $\left.{ }^{4}\right)$; sie würden dann vielleicht um 700 anzusetzen sein. Schon vor langer Zeit hat aber H. De Meulenaere die Gleichsetzung des auf diesen beiden Statuen genannten Wezirs mit dem aus der 3. Zwischenzeit bekannten Wezir P3-mjw bestritten ( $\left.{ }^{5}\right)$ : Zum einen haben sie partiell verschiedene Titel, vor allem der des Dritten Amunpropheten ist nur bei dem P3-mjw der 3. Zwischenzeit belegt; zum anderen sollten die Statuen nach Stil und Inschriften später anzusetzen sein $\left(^{6}\right)$. Außerdem sei Legrains Lesung des Namens des Wezirs auf JE 37413 als P3-mjw sehr zweifelhaft, denn auf Fotos sei die entsprechende Gruppe kaum lesbar; es könne daher ebensogut dagestanden haben.

Die Statue ist vor einigen Jahren von R. el-Sayed veröffentlicht worden $\left({ }^{7}\right)$, und er hat die alte Lesung $P 3-m j w$ bestätigt bzw. übernommen $\left(^{8}\right)$. Allerdings schienen schon die von ihm publizierten Fotos diese Lesung kaum zu bestätigen $\left({ }^{9}\right)$, und tatsächlich ist die Textwiedergabe an vielen Stellen so fehlerhaft, daß sie fast unbrauchbar ist. Im folgenden werden daher die Texte dieser Statue noch einmal insgesamt behandelt, ebenso wie die Statue des Sohnes Kairo JE $37848\left({ }^{10}\right)$. Die Texte von JE 37413 sind in der Tat an verschiedenen Stellen nur mühsam zu entziffern; nach wiederholtem Studium des Originals bleiben aber kaum Fragen offen. Vor allem ist die Lesung des Namens des Wezirs (und Großvaters des Statuenbesitzers) unzweideutig is und nicht (11)

(4) Bei dem im Stammbaum des P3-mjw genannten König handelt es sich um Takeloth III., nicht um Takeloth II., wie A. Leahy eindeutig gezeigt hat (SAK 7, 1979, 148); das ist inzwischen unstrittig, vgl. K.A. KITCHEN, The Third Intermediate Period in Egypt, Warminster ${ }^{2}$ 1986, 597, Table *15; J. YoyotTe, RdE 39, 1988, 169-170; D.A. Aston - J.H. TAYLOR, »The Family of Takeloth III and the 'Theban' Twenty-Third Dynasty « in: A. LEAHY (ed.), Libya and Egypt, London 1990, 132; 140-141; 148.

(5) »L'identité des vizirs Horus et Pimai«, CdE 33, 1958, 194-195.

(6) Nach De Meulenaere gehören sie in den Übergang von 25. zu 26. Dynastie. Bothmer (Egyptian Sculpture of the Late Period, Brooklyn 1960, 5) datiert JE 37413 in die Zeit Psametiks I.

(7) BIFAO 87, 1987, 176-184; pl. XXXII-XXXIV.

(8) Er erwähnt De Meulenaeres Artikel und dessen Zweifel an der Lesung gar nicht.

(9) Vgl. auch J. YoyotTe, $R d E$ 39, 1988, 169, n. 83.

(10) Für die Erlaubnis zur Veröffentlichung und für die Fotos danke ich dem ehemaligen Direktor des Ägyptischen Museums Kairo, Prof. Dr. M. Saleh, sehr herzlich. Nach dem Einreichen des Manuskriptes dieses Artikels ist sie von R. EL-SAYED in: Memnonia 10, 1999, 190-194; pl. LII-LIV publiziert worden. Da seine Textwiedergabe einige kleinere Versehen enthält, ist eine erneute Abschrift dennoch vielleicht nicht ganz überflüssig.

(11) Dieser Wezir $\mathrm{Hr}$ stammt sehr wahrscheinlich aus einer schon in der früheren Dritten Zwischenzeit prominenten Familie, deren wichtigstes Amt das des königlichen Sekretärs von Oberägypten war (»Nb-ntrw-Familie «), und die Hauptlinie der Nachkommen des $\mathrm{Hr}$ (die seine Haupttitel geerbt hat) ist von anderen Statuen her bekannt, vgl. H. DE MEULenAERE, »Le vizir Nebneterou «, BIFAO 86, 1986, 143-149. 
KAIRO JE 37413

\section{Übersetzung}

\section{Oberseite:}

(links) »Gemacht von seinem Sohn, um seinen Namen am Leben zu erhalten, dem Stolisten von Theben, (rechts:) der Recht spricht in Theben (1), der Herrin der Städte, (unten:) der das Geheime des Urzeitlichen der Beiden Länder betritt, des Obersten der Götter (2), 'nh-wn-nfr, gerechtfertigt.«

\section{Vorderseite:}

1 »Der Stolist von Theben, der ḥm-wn (3), der Monatspriester des Hauses des Amun von der 3. Phyle Nb-ntrw, gerechtfertigt, der Sohn 2 des Propheten des Amun in Karnak, des Monatspriesters in der 3. Phyle $\mathrm{h}$ $w n-n f r$, gerechtfertigt, 3 indem er sagt: 'O Propheten und Gottesväter, die in den 'Himmel' eintreten, um den zu sehen, der darin ist, die das Geheime des $\mathbf{4}$ abgeschiedenen Ortes kennen, unter deren Finger die Sonnenstrahlen hervorkommen, um die Erde zu erhellen (4): es belohne euch 5 euer Gott, er liebe euch, indem ihr überflutet seid von Speisen, die er gibt (5), 6 und euer Sohn zur Laufbahn seines Vaters bestimmt ist (6), ohne aufzuhören, seine Reinigung vorzunehmen, 7 so wie ihr eure Arme beugt unter Libationen und Weihrauch.

\section{Rechte Seite:}

1 Möget ihr für mich sprechen 'für seinen Ka die Myrrhe' (7) nach dem Opfern, wenn sich der Gott 2 an seinen Dingen befriedigt hat. Möget ihr sprechen für den Propheten des Month, des Herrn von Theben, Nb-ntrw, gerechtfertigt, 3 'für seinen Ka', wenn seine Speisen mit euch gekommen sind (8). 'Ein anderer wird für uns handeln. Ich (jedenfalls) bin einer mit kühlem Mund' (9). 4 Denn wer handelt, für den wird man das gleiche tun. 5 Eure Hände sollen (daher) nicht leer sein von (zu spendenden) Dingen. Der Atem des Mundes 6 ist wirksam für den Würdigen (Toten), und der Ba kommt auf die Stimme hin.

\section{Linke Seite:}

1 Ein Königsopfer des Amun-Re, des Herrn der Throne der Beiden Länder, des Ersten von Karnak, 2 des Re-Harachte, des Obersten der Götter, 


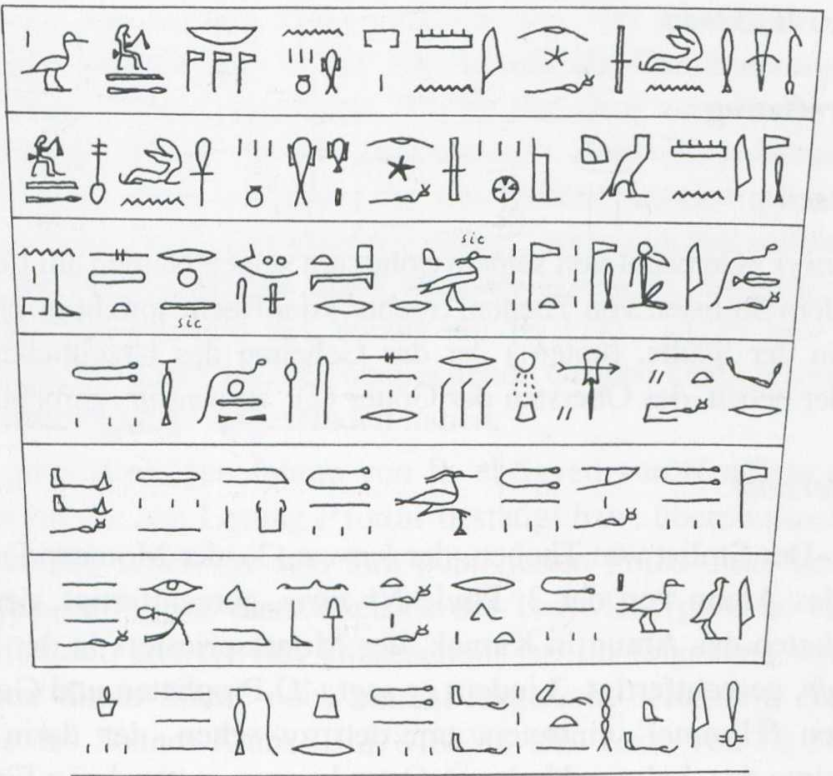

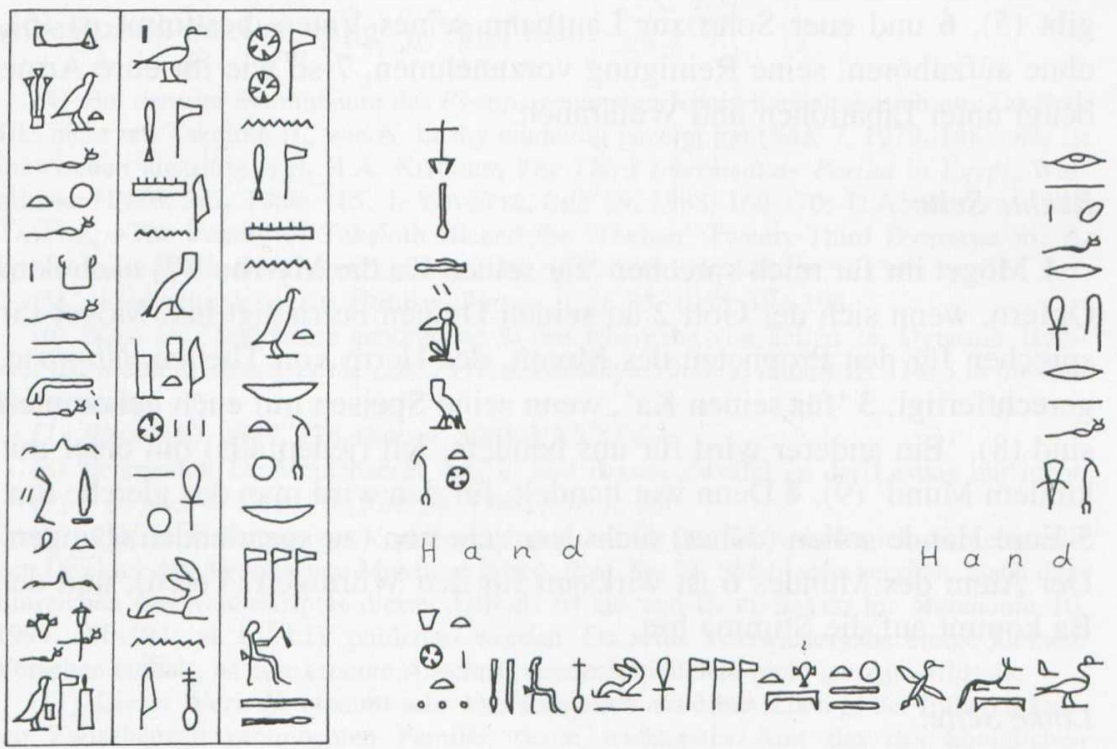

Fig. 1. - Kairo JE 37413. Vorderseite (oben), Oberseite (unten rechts), Rückseite (unten links) 


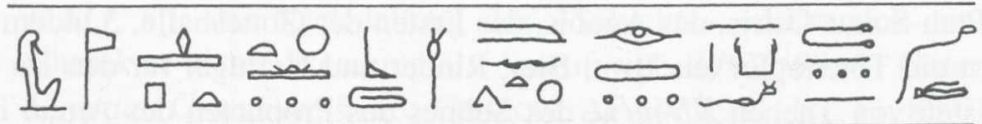

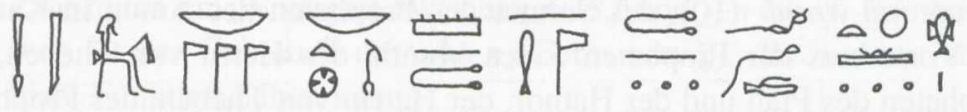

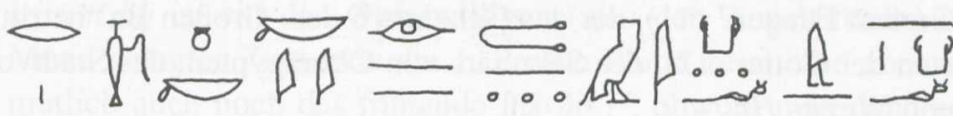

$$
\begin{aligned}
& \text { 4 - }
\end{aligned}
$$

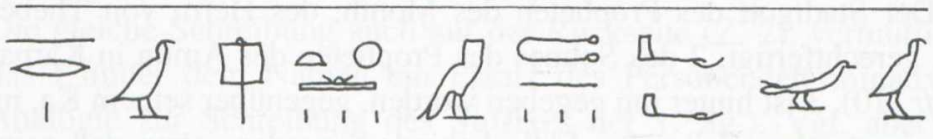

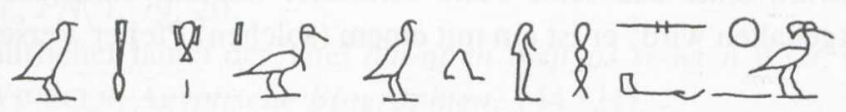

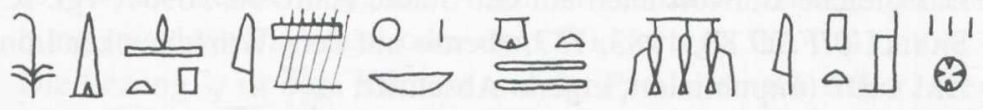

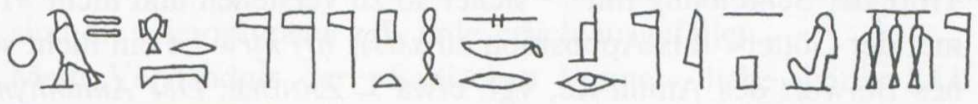

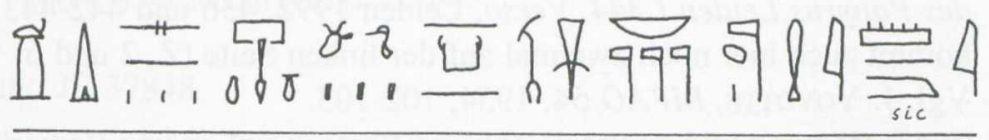

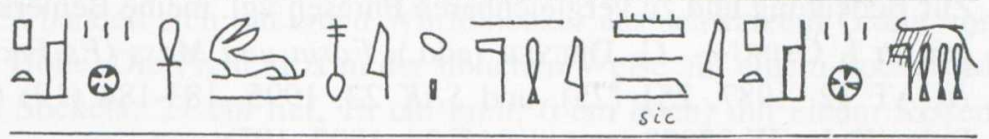

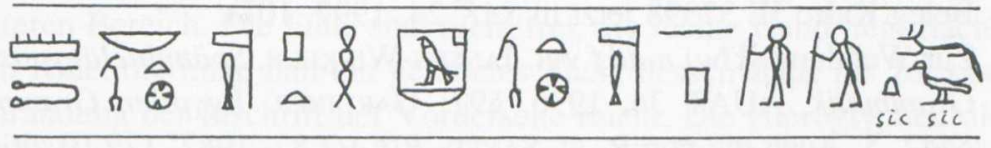

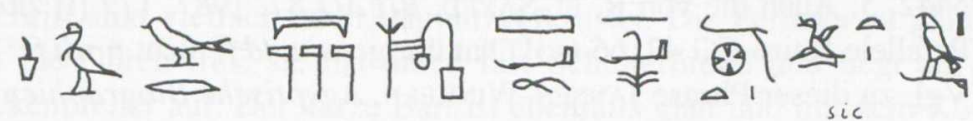
Fig. 2. - Kairo JE 37413. Rechte Seite (oben), Linke Seite (unten) 
des Ptah-Sokar-Osiris, des Anubis, des Ersten der Gotteshalle, 3 indem sie geben ein Totenopfer aus Brot, Bier, Rinder und Geflügel für den Ka des Stolisten von Theben $\mathrm{Nb}$-ntrw, des Sohnes des Propheten des Amun in $\mathbf{4}$ Karnak 'nh-wn-nfr (10), des Sohnes des Propheten des Amun in Karnak, des Vorstehers der Propheten 5 des Month, des Herrn von Theben, des Propheten des Ptah und der Hathor, der Herrin von Theben, des Propheten des 'Großen Fürsten' (11), der das Geheime 6 des 'Großen Ba' betritt, des Obersten der Götter (12), des Sekretärs von Oberägypten, des Stadtvorstehers und Wezirs $\mathrm{Hr}$.

\section{Rückseite:}

1 Der Stadtgott des Propheten des Month, des Herrn von Theben $\mathrm{Nb}$ ntrw, gerechtfertigt, 2 des Sohnes des Propheten des Amun in Karnak $n h$ $w n-n f r(10), 3$ ist hinter ihn gegeben worden, gegenüber seinem $\mathrm{Ka}$, in seine Gegenwart, ohne daß seine Füße behindert werden, ohne daß sein Herz zurückgehalten wird; er ist ein mit einem (solchen) Pfeiler Versehener (13).

\section{Anmerkungen}

1) Das gleiche Beiwort auch auf der Statue Kairo JE 36957, vgl. R. ELSAYED, BIFAO 83, 1983, 137, ebenso auf dem Würfelhocker London BM 1197 (unpubliziert, eigene Abschrift:

2) Trotz der Schreibung mit $\bullet$ sicher so zu verstehen und nicht »Himmel der Götter « (als Apposition zu sšt 3 ); hrj ntrrw ist ein nicht seltenes Beiwort des Amun-Re, vgl. etwa J. ZandeE, Der Amunhymnus des Papyrus Leiden I 344, Verso, Leiden 1992, 436 und 442-443. Es kommt auch hier noch zweimal auf der linken Seite (Z. 2 und 6) vor.

3) Vgl. J. Yoyotтe, BIFAO 54, 1954, 102-103.

4) Zur Bedeutung und zu vergleichbaren Phrasen vgl. meine Bemerkungen in J. Osing - G. Dreyer (edd.), Form und Mass (Fs Fecht), ÄUAT 12, 1987, 251 (25), und $S A K$ 22, 1995, 183-184 (19) (der Beleg Kairo JE 37398 jetzt in SAK 24, 1997, 107).

5) Zur Wendung $(k 3 w) n$ dd.f vgl. JANSEN-WINKELN, Spätmittelägyptische Grammatik, ÄUAT 34, 1996, §97; GARDINER, Egyptian Grammar, $\$ 442$, 5. Auch die von R. EL-SAYED, BIFAO 87, 1987, 179 [i] zitierte Parallele Kairo CG 42166 (so!) hat übrigens $n$ dd.f, nicht $n$ rdj.f.

6) Vgl. zu dieser Phrase JANSEN-WINKELn, Ägyptische Biographien der 22. und 23. Dynastie, ÄUAT 8, 1985, 144-145 (25); 417-418 (5.9.1-2; 7). 
7) $n$ k3.f ${ }^{~} n t j$; die Nachstellung des »Subjekts « erklärt sich aus dem Charakter dieser Wendung als Ausruf, vgl. $W b$ V, 88, 3; GARDINER, Egyptian Grammar, §153; GRAPOW, APAW 1941, Nr. 11, 88-91.

8) Zweifellos ein Wortspiel zwischen $n k 3 . f$ und jj.n k3w.f. Bemerkenswert ist der Gebrauch der sdm.n.f-Form von jjj, die hier nur adverbiale Funktion haben kann.

9) jrj n.n kjj ist ein den Opferwilligen (also den Angerufenen) in den Mund gelegtes Zitat, wie die 1. Person Plural zeigt, und ebenso vermutlich auch noch das folgende jnk $q b r^{e}$, obwohl man dann eigentlich nicht »mit vorsichtigem, ruhigem Mund [= Sprechweise]« erwarten sollte, sondern so etwas wie »der gern bereit ist, (Gebete) zu sprechen «.

10) Die gleiche Schreibung auch auf der Rückseite (Z. 2), vermutlich ist das $i$ hinter dem Namen ein Ersatz des Personendeterminativs (in Analogie zur Schreibung des Suffixes der 1. Sg.). Vgl. aber auch RANKE, $P N, \mathrm{I}, 79,20$.

11) Ausführlicher lautet der Titel hm-ntrr $n$ Wsjr p3 $s r w r n W 3 s t$, s. JANSEN-WINKELN, Ägyptische Biographien, 144 (21).

12) Dasselbe Beiwort auch ibid., 527, Z. 3 (= 394, 4.3.20). »Großer Ba« (b3 wr) als Bezeichnung des Amun ist auch sonst häufig; zur Schreibung mit dem Ba-Vogel oder dem Widder vgl. Wb I, 411 und 414. Die Lesung ${ }^{q} q$ ist dem Kontext und der Parallele nach sicher, aber die Schreibungen am Ende von Z. 5 und 6 sind sehr gedrängt, und daher sind die Hieroglyphen z.T. unleserlich ausgefallen.

13) Mein Verständnis der »Saitischen Formel « habe ich in SAK 28, 2000, 83-124 dargelegt.

\section{KAIRO JE 37848}

Es handelt sich um einen Würfelhocker aus schwarzem Granit von 41 $\mathrm{cm}$ Höhe. Die Figur sitzt in der üblichen Weise auf einem Sockel (Maße des Sockels: $27 \mathrm{~cm}$ tief, $18 \mathrm{~cm}$ breit, $6 \mathrm{~cm}$ hoch) mit einem Kissen im hinteren Bereich. Die Füße sind nicht frei, die rechte Hand liegt flach auf dem Knie, die linke hält ein gefaltetes Tuch, dessen Ende bis zur oberen Umrandung der Inschrift der Vorderseite reicht. Die Hieroglyphen dieser Inschrift sind vielfach noch schmutzverkrustet. Die Perücke ist glatt und läßt die Ohren frei; sie hat unten fast Schulterbreite und liegt auf dem Rückenpfeiler auf. Der kurze Bart ist ebenfalls glatt und mit dem Körperblock verbunden. Auffallend ist die außergewöhnlich niedrige Stirn, die 

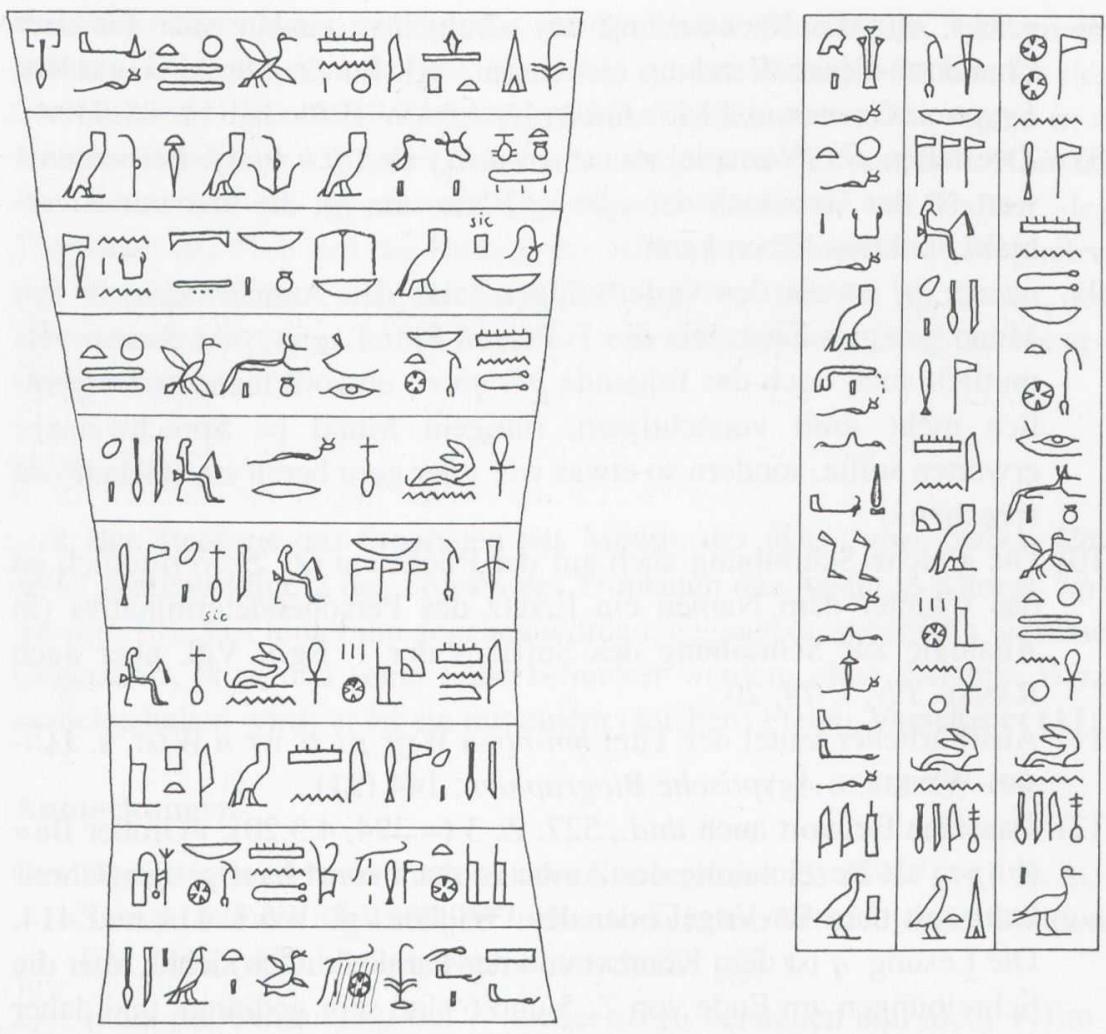

Fig. 3. - Kairo JE 37848. Vorderseite (links), Rückseite (rechts)

sich ebenso bei dem Würfelhocker des Vaters (JE 37413) findet. Auch die Gesichtszüge beider Statuen sind sich sehr ähnlich.

\section{Übersetzung}

\section{Vorderseite}

1 Ein Königsopfer der Amun-Re, des Urzeitlichen der Beiden Länder, indem er gibt ein Totenopfer $\mathbf{2}$ aus Brot, Bier, Rind und Geflügel am Neujahrsfest, am Anfang des Jahres, am $\mathbf{3}$ Wagfest und an jedem Fest des Himmels und der Erde für den Ka des Propheten $\mathbf{4}$ des Month, des Herrn von Theben, der das Geheime des Urzeitlichen der Beiden Länder sieht, 5 $n h$ $w n-n f r$, gerechtfertigt, des Sohnes $\mathbf{6}$ des Stolisten von Theben Nb-ntrw, 
gerechtfertigt, des Sohnes des Propheten 7 des Amun in Karnak $n h$-wn-nfr, 8 gerechtfertigt, des Sohnes des Propheten des Amun in Karnak 9 und Vorstehers der Propheten des Month, des Herrn von Theben, 10 des Sekretärs von Oberägypten, Stadtvorstehers und Wezirs $H r$, gerechtfertigt.

\section{Rückseite}

1 Der Stadtgott des Propheten des Month, des Herrn von Theben, der das Geheime des Urzeitlichen der Beiden Länder sieht, " $n h$-wn-nfr, gerechtfertigt, des Sohnes 2 des Stolisten von Theben Nb-ntrw, gerechtfertigt, des Sohnes des Propheten des Amun in Karnak 'nh-wn-nfr (1), gerechtfertigt, ist gesetzt worden 3 hinter ihn, gegenüber seinem Ka, in seine Gegenwart, ohne daß seine Füße behindert werden, ohne daß seine Arme zurückgehalten werden; er ist ein mit einem (solchen) Pfeiler Versehener (2).

\section{Anmerkungen}

1) Zur Schreibung des Namens s.o., Anm. 10 zu JE 37413.

2) Zur »Saitischen Formel« s.o., Anm. 13.

\section{Nachträge Zur Statue Kairo JE $37196\left({ }^{12}\right)$.}

Dieser Würfelhocker ist 1976 von E. Bresciani veröffentlicht worden ${ }^{13}$ ). Allerdings ist die Wiedergabe des Textes (der tatsächlich auch recht mühsam zu lesen ist und wiederholte Kollationen erforderlich machte) keineswegs fehlerfrei; vor allem der biographische Abschnitt auf der Vorderseite ist weitgehend mißverstanden worden. Eine neue Abschrift ist daher sicher nicht überflüssig. Außerdem ist die Datierung der Statue problematisch. Bresciani hatte sie in die 25. Dynastie datiert $\left({ }^{14}\right)$; der frühest mögliche Zeitpunkt sei das Ende der 23. Dynastie (der Amtsbeginn der Gottesgemahlin Schepenupet I., deren Namen in einem Titel erscheint), der spätest mögliche aus stilistischen Gründen die Regierungszeit Psametiks I. Diese stilistischen Kriterien $\left({ }^{15}\right)$ sind die Art der Perücke sowie das Gewand und die Modellierung von Armen und Beinen. Die "zweiteilige « glatte Perücke, ohne Angabe von Haarsträhnen, ist nach

(12) Ich danke Herrn Prof. Dr. H. De Meulenaere herzlich für zahlreiche wertvolle Hinweise.

(13) Studi classici e orientali 25, 13-21.

(14) Op. cit., 18-21.

(15) Op. cit., 18, n. 1. 
Bothmer $\left({ }^{16}\right)$ auf die 25. Dynastie und die Zeit Psametiks I. beschränkt. Tatsächlich ist es aber gar nicht sicher, ob die Figur überhaupt eine zweiteilige Perücke trägt: Der untere Teil des Kopfes ist handwerklich so schlecht gemacht, daß diese Frage nicht ganz sicher zu entscheiden ist. Es könnte sich auch um eine einfache glatte Perücke handeln, die für die Datierung nichts hergibt. Für das zweite Datierungskriterium beruft sich Bresciani ebenso auf Bothmer $\left({ }^{17}\right)$, der gesagt hat, daß Würfelhocker » with short loincloth, feet uncovered, and arms modeled nearly in the round « in der 25. Dynastie häufig seien, aber gegen Ende der Regierung Psametiks I. verschwänden. Auch dieses Kriterium trifft für JE 37196 nicht zu: Die Arme sind keineswegs »modeled nearly in the round «, und Würfelhocker mit unbedeckten Füßen sind auch nach Psametik I. durchaus üblich $\left({ }^{18}\right)$. B.V. Bothmer zufolge gehört die Statue tatsächlich in nachpersische Zeit. In den Unterlagen des Corpus of Late Egyptian Sculpture heißt es: »PostPersian, but rather unusual inasmuch as the wig covers the ears partly which, otherwise, only occurs to the end of Psametik I « (zitiert nach brieflicher Mitteilung von H. De Meulenaere). Prof. De Meulenaere bevorzugt dagegen eine Datierung ins 7. Jahrhundert $\left({ }^{19}\right)$, nicht zuletzt aufgrund der Tatsache, daß ein Kult des Osiris $h q 3 \underline{d} \underline{d}$ nach der 26. Dynastie nicht mehr belegt ist, ebensowenig dieser Beiname. Auch der Titel jmj-st- ${ }^{\circ} n p 3$ htp 3 $w^{c} b n$ Jmn taucht in nachsaitischer Zeit nur noch ganz vereinzelt auf $\left({ }^{20}\right)$. Dennoch scheint mir angesichts der recht nachlässigen Arbeit und Beschriftung dieser thebanischen Statue eine Datierung in die 25. oder 26. Dynastie schwer vorstellbar und ein Ansatz in das 4. Jahrhundert wahrscheinlicher.

\section{Übersetzung}

\section{Vorderseite}

1 Ein Königsopfer des Amun-Re, des Herrn der Throne der Beiden Länder, des Herrn des Himmels, Herrschers von Theben, (bestehend

(16) Egyptian Sculpture of the Late Period, 3.

(17) Egyptian Sculpture of the Late Period, 37-38.

(18) Vgl. etwa ibid., pl. 73.

(19) Die Bemerkung bei E. GRAEFE, Untersuchungen zur Verwaltung und Geschichte der Institution der Gottesgemahlin des Amun vom Beginn des Neuen Reiches bis zur Spätzeit, ÄA 37, 1981, Bd. II, 67, n. 227, ist so nicht richtig, wie mir Prof. De Meulenaere mitteilt.

(20) S. J. QuAEgEBEuR, RdE 45, 1994, 155-173 (einziger nachsaitischer Beleg Turin 3070). 
aus) allem, was 2 von seinem Altar kommt und allem, was auf seinem Opfertisch dargebracht wird an allen Anfangsfesten der Zeitläufe, für den $\mathrm{Ka}$ des Gottesvaters und $m r j j(-n t r)$, des Schreibers des Vorhofes 3 des Hauses des Amun und seiner Heiligtümer (1) $D d-H n z w-j w . f-{ }^{-} n h$, gerechtfertigt, des Sohnes des gleichrangigen $4 J w . f-3$, gerechtfertigt, geboren von der Hausherrin und Musikerin des Amun-Re Ns-nb- $n h(w)$ (2), gerechtfertigt, indem er sagt: $5 \mathrm{O}$ mein Herr, o mein Herr, ich bin ein Diener mit gerechtem Herzen, ein Aufrichtiger, der sich nicht zu dem Schuldbeladenen gesellt (3), der zufrieden ist über die Korrektheit (4), ohne unaufmerksam zu sein, wenn mein Gesicht in deiner Einsamkeit gewesen war(?) (5), der Sohn eines Dieners deines Hauses (6).

\section{Rückseite}

1 Der Stadtgott des Gottesvaters und $m r j j(-n t r)$, des Propheten des Osiris »Herrscher der Ewigkeit « der Gottesanbeterin $\breve{S} p$ - $n$-wpt (7), der $j r j-\check{s} n$ des Hauses des Chons in Theben, Neferhotep, in der 2. Phyle (8), 2 der $\underline{t} 3 y$-Schreiber des Hauses des Amun von der 1. Phyle (9), der diensttuende Priester des großen reinen Opfertisches des Amun (10) des Götterbildes(?) und der Station (11) der 2. Phyle und in der 4. Phyle, 3 der große Wab-Priester des Amun in der 4. Phyle $\underline{D} d-H n z w-j w . f-i n h$, gerechtfertigt, der Sohn des gleichrangigen $J w . f_{-}^{-} 3$, gerechtfertigt, ist hinter ihn gegeben worden, gegenüber seinem Ka, in seine Gegenwart; er ist mit einem (solchen) Pfeiler Versehener (12).

\section{Um den Sockel}

1 Ein Königsopfer des Amun-Re, des Herrn des Himmels, Herrschers von Theben, indem er gibt ein Totenopfer aus Brot, Bier, Rind, Geflügel und allen guten und reinen Dingen für den Ka des Gottesvaters und $m r j j$ $(-n t r)$, des Propheten des Osiris »Herrscher der Ewigkeit « der Gottesanbeterin $\breve{S} p$ - $n$-wpt (7), des $j r j-\check{s} n$ des Hauses des Chons in Theben in der 2. Phyle (8), des $t 3 y$-Schreibers 2 des Hauses des Amun von der 1. Phyle (9), des diensttuenden Priesters des großen reinen Opfertisches des Amun (10) des Götterbildes(?) und der Station (11) der 2. Phyle und in der 4. Phyle, des großen Wab-Priester des Amun in der 4. Phyle, des Schreibers des Vorhofes des Hauses des Amun und seiner Heiligtümer (1) $D d-H n z w-j w . f-i h$, gerechtfertigt, des Sohnes des gleichrangigen $J w . f^{-}{ }^{-3}$, gerechtfertigt. 

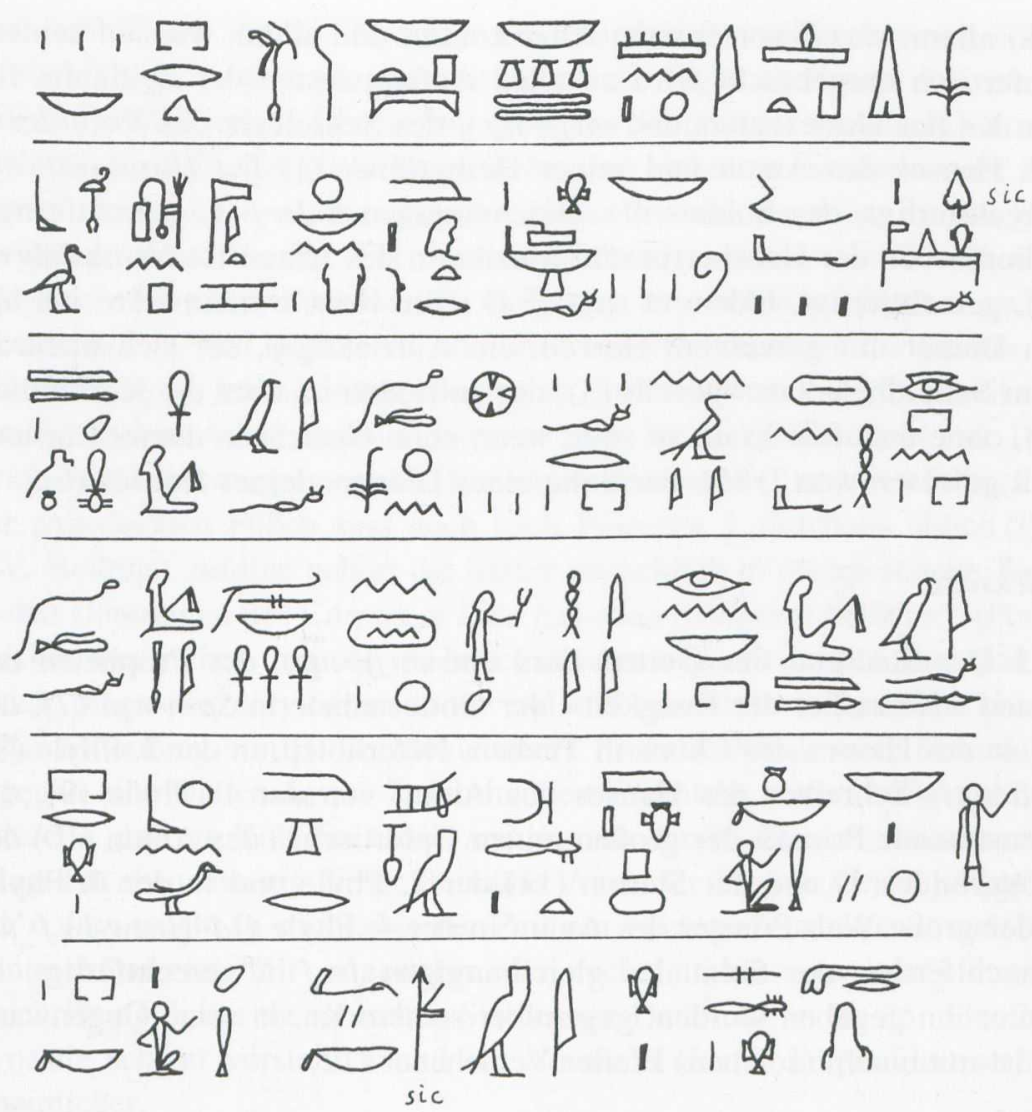

Fig. 4. - Kairo JE 37196. Vorderseite

\section{Anmerkungen}

1) Der Titel $z \check{s} n$ p 3 wb3 ( $n$ pr Jmn) hat mit der Rechtsprechung am Tempeltor zu tun, vgl. zuletzt J. QuaEgeBeur, in: Ch. CANNUYeR - J.-M. KRUCHTEN, Individu, société et spiritualité dans l'Égypte pharaonique et copte (Mélanges A. Theodoridès), 201-220 (bes. 203-204). Der Zusatz $h n^{c} n 3 j$.f $r 3 w$-prw findet sich manchmal auch bei dem Titel $z \check{s}$ hwt-ntr n Jmn, s. J.-Cl. GoYON, Karnak VII, 283 (G). Er bestätigt indirekt, daß es sich nicht um einen eigentlichen Priestertitel handelt, denn die Verwendung des Possessivartikels deutet auf einen »profanen « Tätigkeitsbereich, vgl. Jansen-Winkeln, Text und Sprache in der 3. Zwischenzeit, ÄUAT 26, 1994, 226-7. 

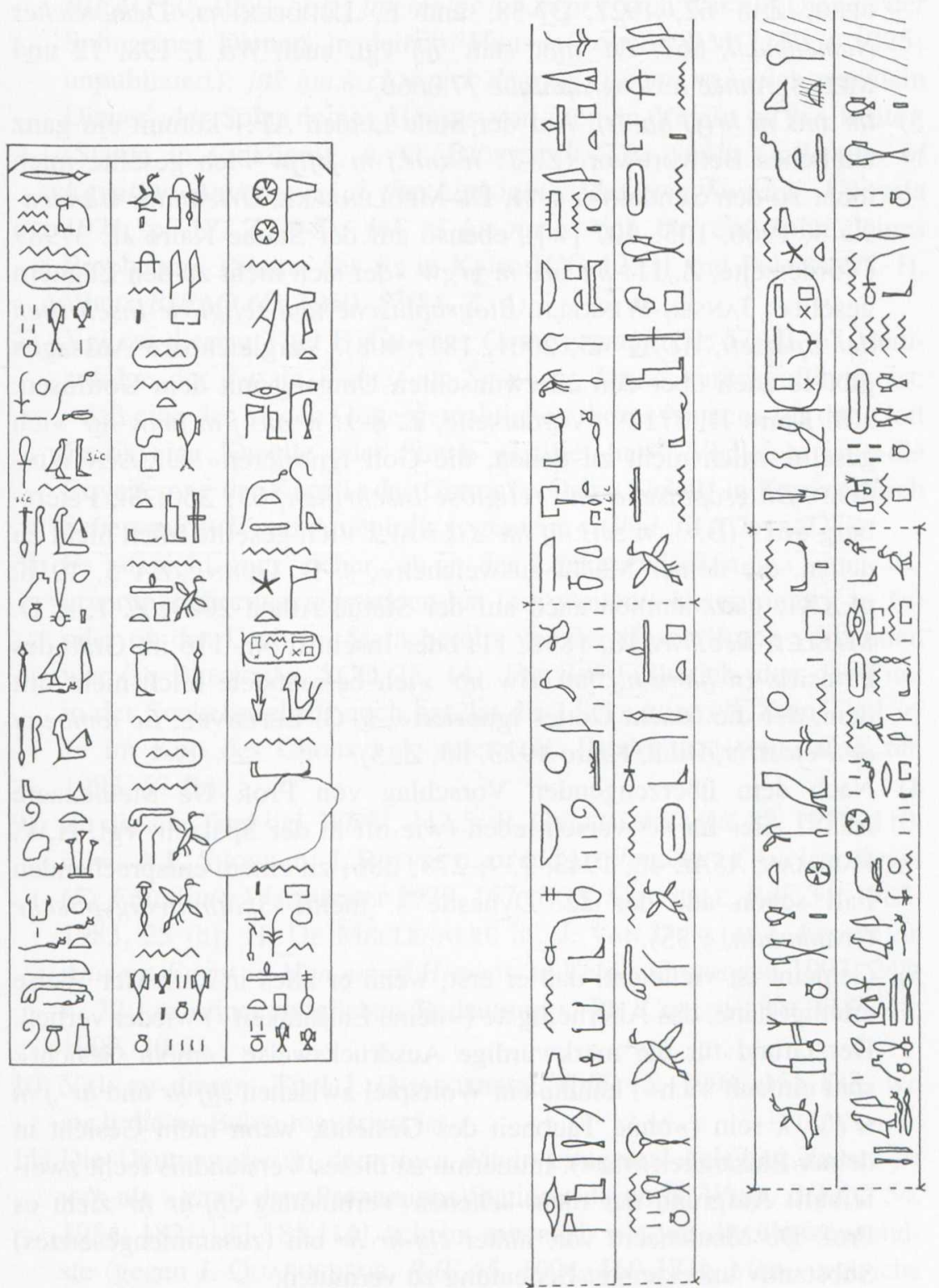

Fig. 5. - Kairo JE 37196. Rückseite (links), Sockel (rechts) 
2) Die übliche Form dieses Namens ist $N s-(p 3-) n b-{ }^{-} n h$, s. W. SPIEGELBERG, ZÄS 62, 1927, 37-38, und E. LüDDECKENS, Demotisches Namenbuch, 667. Zu 'nhw statt 'nh vgl. auch Wb I, 198, 12 und MEEKs, Année lexicographique 77.0666.

3) $t m z m 3 m h r(j) h b n(t)$. Auf der Stele Leiden AP.1 kommt ein ganz ähnliches Beiwort vor (Z. 7: $n$ zm3.j $m$ jsftjw »ich gesellte mich nicht zu den Sündern «, s. H. De Meulenaere, Orientalia Gandensia 3, 1966, 105; 107 [w]), ebenso auf der Statue Kairo JE 37989 (Vorderseite, Z. 11: $\mathrm{tm} \mathrm{zm} 3 \mathrm{~m}$ grgw »der sich nicht zu den Lügnern gesellt «; JANSEN-WINKELN, Biographische und religiöse Inschriften der Spätzeit, $\ddot{A} U A T$ 45, 2001, 181; 408). Vergleichbare Aussagen gibt es auch über den unerwünschten Umgang mit dem Gottlosen, z.B. Kairo JE 37129 (Vorderseite, Z. 8-9: $n$ zm3.j $m$ hymw ntr »ich gesellte mich nicht zu denen, die Gott ignorieren «: JANSEN-WINKELN, Biographische und religiöse Inschriften, 65; 360; St. Petersburg 5629 (B, 3: $n$ zm3.n.j ḥn ${ }^{e} \breve{3} 3 \check{s} b 3 w . k$ »ich gesellte mich nicht zu denen, die deiner Macht ausweichen«, s. B. GunN, JEA 5, 1918, pl.XXI; ganz ähnlich auch auf der Statue Athen 2009, Z. 7, s. D. Mallet, RecTrav 18, 1896, 11) oder Inschrift Nr. 116 im Grab des Petosiris ( $n$ hnms.n.j hm b3w ntr »ich befreundete mich nicht mit dem, der die Macht Gottes ignoriert «, s. G. LeFEbvre, Le tombeau de Pétosiris, Bd.2, Kairo 1923, 83, Z. 5).

4) Nach dem überzeugenden Vorschlag von Prof. De Meulenaere ist $\square$ hier für $\square$ verschrieben (wie oft in der Spätzeit, vgl. H.W. FAIRMAN, ASAE 43, 1943, 239; 278; 286; zu einem entsprechenden Fall schon aus der 22. Dynastie s. meine Spätmittelägyptische Grammatik, § 35).

5) Gemeint ist vielleicht, daß er erst, wenn er alles in korrekter Weise erledigt hatte, das Allerheiligste (»deine Einsamkeit «) wieder verließ. Der Grund für die merkwürdige Ausdrucksweise (»mein Gesicht « statt einfach »ich«) könnte ein Wortspiel zwischen zh̆j ḥr und $h r-j m$ $w^{c}\left({ }^{e} w\right) . k$ sein (»ohne Taubheit des Gesichts, wenn mein Gesicht in deiner Einsamkeit war«). Immerhin ist dieses Verständnis recht zweifelhaft. Aufgrund der nicht seltenen Verbindung zhjj-ḥr ḩr zieht es Prof. De Meulenaere vor, hinter $z h j$ - $h r h h r$ ein (zusammengesetztes) Substantiv unbekannter Bedeutung zu vermuten.

6) Entsprechende Formulierungen sind nicht selten, z.B. jnk ḥm z3 ḥm $n$ pr pn »ich war ein Diener, der Sohn eines Dieners dieses Hauses « (Kairo CG 672, s. R. EL-SAYED, Documents relatifs à Saïs et ses 
divinités, BdE 69, 1975, 86; Marseille 214 [zitiert bei H. WILD, BIFAO 60, 1960, 56]); jnk hm z3 hm $m$ pr.t »ich war ein Diener, der Sohn eines Dieners in deinem Haus « (London BM $1132+1225$; unpubliziert); jnk hmm.k z3 n pr.k $\underline{d} r j t j w(. j) r-m n$ mjn »ich war dein Diener, der Sohn deines Hauses von meinen Vätern an bis heute « (Statue in Linköping, s. G. BJöRKMAN, The Smith Collection of Egyptian Antiquities at the Linköping Museum, Sweden, Uppsala 1971, p. 17, Z. 4-5); jnk z3 hm-ntr.k »ich bin ein Sohn deines Propheten « (Statue des Bs in Kairo [CG 1233] und Palermo, s. H. WILD, BIFAO 60, 1960, 53/54, Z. 4).

7) Vgl. zu diesem Titel E. GRAEFE, Gottesgemahlin, I, 57; II, 67, demzufolge der Zusatz $n d w 3 t-n t r$ r $\breve{S}$ - $n$-wpt darauf zurückzuführen ist, »daß eine der beiden Gottesgemahlinnen dieses Namens für den Gott Osiris eine Kapelle oder Statue gestiftet hat «. Vielleicht ist »die Erweiterung der Kapelle des Gottes [= Osiris $h q\} \underline{d} t]$ in Karnak durch Schepenupet I. und Amenirdis I. gemeint (ibid., II, 67, n. 227).

8) Es ist nicht ganz sicher, ob in der Lücke zwischen $\leftrightharpoons$ und mm ursprünglich etwas gestanden hat (ein weiteres Determinativ zu šn) oder ob das Loch im Stein bereits vor der Beschriftung vorhanden war (so BRESCIANI, SCO 25, 14). Für den Füllstrich, den der Titel in der Sockelinschrift noch hat, ist die Lücke zu groß. Zum Titel jrj $\check{s} n$ im Kult des Chons vgl. zuletzt H. De Meulenaere, CdE 68, 1993, 57 (b).

9) Zu diesem Titel vgl. $W b$ V, 342,5; E. GraEFe, MDAIK 35, 1979, 110, n. w; A.F. SHORE in: J. Ruffle u.a. (edd.), Glimpses of Ancient Egypt (Fs Fairman), Warminster 1979, 157 (f); S. CAUVILLE, RdE 34, $1982-$ 1983, 25 (h); H. De Meulenaere in: J. van DiJK (ed.), Essays on Ancient Egypt in Honour of Herman te Velde, Groningen 1997, 247, n. 32; zu seiner möglichen Bedeutung s. Ph. Collombert, $R d E$ 48, 1997, 20-21.

10) Vgl. zu diesem Titel J. Quaegebeur, $R d E$ 45, 1994, 155-173, wo auch dieser Beleg registriert ist.

11) Die Deutung des in derartigen Titeln einigemal belegten Zusatz $p 3$ w 3 h als »(von) der (Prozessions)Station « durch H. WILD, BIFAO 54, 1954, 182; 183-185 [14], scheint mir nach wie vor die überzeugendste (gegen J. QuAEGEBEUR, $R d E$ 45, 1994, 169-171). Man vergleiche auch P. BARGUET, Le temple d'Amon-Rê à Karnak, Kairo 1962, 10, n. 1 , mit einem Beleg von einer Barkenstation. Der (seltenere) Zusatz ( $n$ ) p3 mtr (vgl. QuAEGEBEUR, loc. cit.) müßte analog zu verstehen sein. In 
Kom Ombo ist eine Bezeichnung des Kultbildes (o.ä.) als p3 mtr belegt (vgl. Wb II, 174,5). Zusammen könnte $n$ p3 mtr p3 w3h dann bedeuten, daß er sowohl am (stationären) Götterbild als auch bei der Prozession der Barke Opferdienst tat. Allerdings scheint ein Titel wie $m r \check{s} n(n)$ p 3 w $3 h$ (Kairo CG 995, Rückseite, 2) mit diesem Verständnis kaum erklärbar zu sein.

12) $\mathrm{Zu}$ diesem Verständnis der »Saitischen Formel « s. meinen Artikel in SAK 28, 2000, 83-124.

Karl JANSEN-WINKELN 